\title{
Cervical Spondylotic Radiculopathy: The Role of Conservative Management
}

See Lei Peng ${ }^{1}$, Zamzuri Bin Zakaria@Mohomad", Rajandra Kumar a/l Karupiah", Marazuki bin Hj Perwira ${ }^{2}$

${ }^{1}$ Department of Orthopaedics, Traumatology and Rehabilitation, Kulliyyah of Medicine, International Islamic University Malaysia

${ }^{2}$ Department of Orthopaedics, Hospital Kuala Lumpur

Presenter: See Lei Peng

Introduction: Cervical spondylotic radiculopathy is common and most patients experiencing an acute episode of unilateral radiculopathy without major motor deficit and no evidence of cord compression can be managed by non-operative measures. This study aimed to determine the severity of degenerative changes on plain radiograph among patients with cervical spondylotic radiculopathy and patient's self rated outcomes following conservative treatment. This study also looked into the correlation between pain with disability of patient, severity of degenerative changes of the cervical spine and different level of root compression. Materials and Methods: An observational cohort study where all patients with newly diagnosed cervical spondylotic radiculopathy were recruited from December 2016 to January 2018. Patient rated pain score and neck disability index at presentation and three months after conservative treatment were evaluated. Demographic data, radiographic severity grade, neck disability index and pain score were documented and analyzed via descriptive analysis and Pearson correlation test. Results: There were 29 patients recruited, with mean age of 49 years old. Forty one percent of patients had Grade 2 radiographic severity changes. Patient rated outcomes with neck disability index and pain score showed moderate improvement after conservative treatment $(r=0.523, p<0.01)$. There was significant correlation between pain score and neck disability index $(p<0.001)$. However, pain score showed no significant correlation between radiographic severity grade and different level nerve root compression. The power of this study is 0.8 with PASS 11 calculation. Conclusion: Patients with cervical spondylotic radiculopathy who were treated conservatively showed favorable clinical outcome. 